\title{
ASSESSING MOTORCYCLIST PROTECTION SYSTEMS USING FINITE ELEMENT SIMULATIONS
}

\author{
Mantaras, D. A. \& Luque, P. \\ Ingeniería e Infraestructura de los Transportes, Universidad de Oviedo - Campus de Gijón 33203, \\ Spain \\ E-Mail: luque@uniovi.es
}

\begin{abstract}
The impact of motorcyclists against the posts of the roadside barriers is one of the most frequently and harmful accidents. In order to avoid or minimize impact effects, different safety systems are being installed in many roads in the world. These safety systems should conform technical standards. European Technical Specification 1317-8 defines how it should be the testing procedure and placement of systems to prevent such accidents. The full-scale crash test with a dummy requires certain values not exceeded in neck forces, moments and Head Injury Criterion (HIC). This paper applies computer simulations (using Finite Element Method) in order to develop a virtual testing program to assess the safety system and evaluate the weaknesses of the mentioned standard. (Received in April 2014, accepted in July 2014. This paper was with the authors 1 month for 1 revision.)
\end{abstract}

Key Words: Finite Element, Motorcyclist Protection, Roadside Safety

\section{INTRODUCTION}

Motorcyclists or Powered Two-Wheelers (PTW) are vulnerable road users, their injury rates are about 7 times higher than rates of other motor vehicle occupants and the risk of death is about 20 times higher than from other motor vehicles [1]. Prevention of motorcycle crashes will result in improvement in overall road traffic safety and significant reduction in economic losses [2]. To develop proper countermeasures different studies and databases [3] have been developed in order to analyse of the causes and consequences of motorcycle accidents [4-6].

In the event of a run-off accident, they have a high risk of suffering critical interaction with hazardous obstacles placed on the roadsides, including safety barriers [7-11]. Due to these impacts, the riders are more likely to suffer injuries to upper and lower extremities, and vital regions of the body, such as the spine, head and thorax [3, 12-13]. To address this safety issue, roadsides are equipped with so-called "Motorcyclist Protection Systems" (MPS) [14].

A widely followed approach to reduce potential injury hazards is to prevent contact with geometries that could potentially concentrate impact forces on the human body. This idea led for instance to develop additional lower rails and of absorbing envelopes for the metal barrier posts. The development of safety devices needs tools capable of predicting the injury risk and of evaluating the protection of road users (car occupants, pedestrians, two wheels users). For a long time, mechanical crash test dummies have only represented these tools. They were used for car safety research, development, and regulatory testing as well.

Nowadays, the safety performance of MPSs are assessed by performing crash tests using anthropomorphic test devices (i.e. crash test dummies). Several crash test procedures have been developed in different countries $[15,16]$. In Europe, three main test protocols are in use (LIER, UNE135900, BASt), although the first standard in this field was the Spanish standard UNE 135900-2005 [17]. This standard became a reference for the development of new protection systems to be installed on roads. In January 2012, the countries represented at CEN (Centre Européen de Normalisation) approved a technical specification (TS) 1317-8 for the testing of the impact severity of safety barrier [18]. The protocol of the TS 1317-8 is virtually the same as the Spanish standard UNE 135900. Among the existing protocols, the way to test 
the MPS (Motorcyclist Protection System) is very similar (except the German one for upright impacts). Basically, the testing procedure simulates a motorcyclist sliding (after separation from the powered two-wheelers) on the road at high velocities (above $50 \mathrm{~km} / \mathrm{h}$ ), without the motorcycle, and crashing into the MPS.

\subsection{European regulation for the roadside motorcyclist protective devices}

The purpose of this standard is to define the methods that allow evaluating the behaviour of the motorcyclist protection systems (MPS), punctual as well as continuous ones. The full safety barrier has 60 meters of length consisting of 15 posts at 4 meters spacing. In the beginning and end, the guardrail was dipped into the ground over a distance of 12 meters, consisting of six poles at 2.0 meters spacing. This gives the total guardrail length 80 meters. The requirement of this procedure is that a modified Hybrid III $50^{\text {th }}$ percentile male dummy, sliding on the floor, impacts the protection system to be tested, with a specific angle and speed. The dummy must to be equipped with an integral helmet, a leather motorcyclist suit and a cotton shirt. Depending on the kind of system to be tested, a different trajectory (Fig. 1) is chosen: centred post impact, eccentric post impact or centred rail impact.

The standard defines the impact speeds of $60 \mathrm{~km} / \mathrm{h}$ or $70 \mathrm{~km} / \mathrm{h}$. In order to get the required speed and trajectory the dummy is put on a trolley that is guided with the desired speed and direction. Few meters before the point of impact the trolley stops and the dummy falls out of it and sliding on the floor. In this way, during the approach path the dummy bounces slightly while the neck rotates. The vertical movements of the dummy and the neck rotation makes the impact height varies slightly from one test to another. The standard does not admit a deviation of more than $60 \mathrm{~mm}$ between the actual point of impact and the theoretical point of impact measured on the longitudinal alignment, but do not set any limit deviation on the height of it.

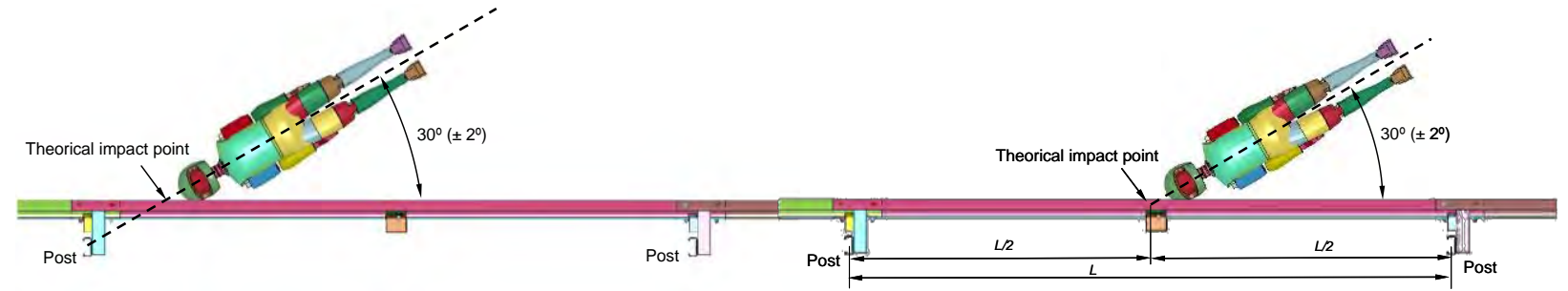

Figure 1: Two different impact points: straight into the posts and between two posts.

The main objective of the roadside barriers is to avoid the impact of the sliding motorcyclist with the barrier post and, at same time, to redirect the motorcyclist into de road but very close to the barrier, both of them with a limited impact severity. In order to evaluate the impact severity of the barrier, the following measurements have to be taken with the instrumented dummy:

- The head injury criteria (HIC36),

- The neck forces and moment at the occipital condyle.

Table I: Maximum values of dummy measurement for the two class of severity [18].

\begin{tabular}{|c|c|c|c|c|c|c|c|}
\cline { 2 - 7 } \multicolumn{1}{c|}{} & $H I C_{36}$ & $\begin{array}{c}F_{x} \\
(\mathrm{~N})\end{array}$ & $\begin{array}{c}F_{z} \text { traction } \\
(\mathrm{N})\end{array}$ & $\begin{array}{c}F_{z} \text { compression } \\
(\mathrm{N})\end{array}$ & $\begin{array}{c}M_{x} \\
(\mathrm{Nm})\end{array}$ & $\begin{array}{c}M_{y} \text { extension } \\
(\mathrm{Nm})\end{array}$ & $\begin{array}{c}M_{y} \text { flexion } \\
(\mathrm{Nm})\end{array}$ \\
\hline Level I & 650 & Diagram & Diagram & Diagram & 134 & 42 & 190 \\
\hline Level II & 1000 & Diagram & Diagram & Diagram & 134 & 57 & 190 \\
\hline
\end{tabular}

From the measurements, two severity classes (Table I) are established, depending on biomechanical criteria, level I and II. After a test, if the system does not meet the minimum 
requirements in the worst of the two levels, level II, this barrier is not considered suitable for installation on the roads.

As exposed, the standard describes the procedure of what simulates the impact of a motorcyclist at a certain speed, in two areas of the barrier and with a certain angle of approach. Obviously, it does not represent all type of possible impacts but the test protocol of a standard must be an objective reference to establish the minimum safety level of a new device. Some of the factors that have more influence in the neck forces and in the head acceleration are the geometry and type of helmet (trade mark for example). For this reason, the standard defines a reference crash helmet (trade mark, model and size). Also in this case, it does not represent all type of possible helmets but if all tests are made with the same type of helmet the objectivity of the results is assured.

Other factors that have significant influence in the neck forces and in the head acceleration are the impact conditions like approach speed, longitudinal position of the impact point and the angle of approach. This is supported by analyses of different authors. The influence of approach angle and speed, in numerical crash scenario according to technical standard, was evaluated using a multi-body dummy [19]. Other authors have evaluated different barrier types [20]. It has been demonstrated that a rise in the impact speed causes an increase in all the measured physical values in the virtual dummy and that the higher the impact angle, more serious are the injuries provoked by the impact [16]. Due to this, the existing protocols (Spanish standard for example) provide a speed and an angle of approach with a small limit deviation. Furthermore, this standard establishes limit deviations for longitudinal position of the impact point.

However, the existing protocols do not assess the influence of other physical parameters. After examining high-speed video recordings of real tests, an important (and close to random) deviation was noticed in the height point of impact. Due to the geometry of the dummy and helmet when the dummy is lying on its back, a clearance appears between helmet and sliding surface (loose gravel). When the dummy is moving towards the MPS, the head is bouncing and rebounding, and the neck changes its angular position. This deviation in angular position modifies the height of impact point. The total deviation of this value can reach values of $45 \mathrm{~mm}$.

This parameter affects to the contact geometry and is supposed that could have a relevant role in the crash mechanics and injuries causation. After a detailed examination of the mentioned testing protocols, can be asserted that the existing testing protocols include no mention related to deviation limits neither procedure to measure or fix the height of impact point to deviation limits or acceptable intervals.

In this work, an analysis of its influence was put forward, by means of numerical model based in Finite Element Method (FEM). This methodology shows how the height of the point of impact significantly influences the behaviour of the system, changing the impact severity level and the potential injury.

\section{VIRTUAL TESTS PROGRAM METHODOLOGY}

The present methodology, to show how the height of the point of impact significantly influences the behaviour of the system, was developed in three steps. In the first step, a detailed finite element model of the protection system and the motorcyclist was created incorporating details of the dummy and helmet, standard rail, protective device, posts, bolts, connections and the soil in which the posts were embedded. The second step involved fit and validations of the model by comparisons of simulated results to data from full-scale crash tests. The third step involved using the validated model as the basis for studying the effects of the impact location height. 
In this work, the dynamic explicit finite element code LS-DYNA® [21] was used to simulate the crash performance of the roadside motorcyclist protective devices in accordance with procedures of the regulation. The LS-DYNA finite element analysis program uses an explicit Lagrangian numerical method to solve three-dimensional, dynamic, nonlinear, large displacement problems.

\subsection{The virtual test model}

A full finite element model of the protection system and the motorcyclist was implemented. To simulate the motorcyclist, a deformable finite element model of the Hybrid III $50^{\text {th }} \%$ dummy was used. The dummy model used in this work has been developed by LSTC. LSTC produce a range of freely available dummy models that are suitable for basic loading analysis [21]. The dummy model has been modified to comply with the regulation requirements. A new geometry of the clavicle and hip was used and the position of the legs and neck was modified. The helmet is fixed to the head by the retention system and a contact between the head and inside part was defined. The final appearance is shown in Fig. 2 a.

The helmet model (Fig. 2 b) has two main parts, a rigid outer shell and an energy absorbing liner $[22,23]$. The outside, which prevents the penetration of sharp objects, is made of thermoplastic material. The protective padding is mainly made of expanded polystyrene foam, which prevents injury to the head during impact. In the chin part it is not used an absorbent material, and it was modelled as comfort padding.

To carry out the virtual model, a three-dimensional scanning of the inner and outer surfaces of the helmet was performed, obtaining a digitized model of it. The outer part was meshed with 1882 shell type elements, while the interior was meshed with 5646 solid elements. The helmet finite element model has 7652 nodes. The materials used in the different parts are shown in the table II.
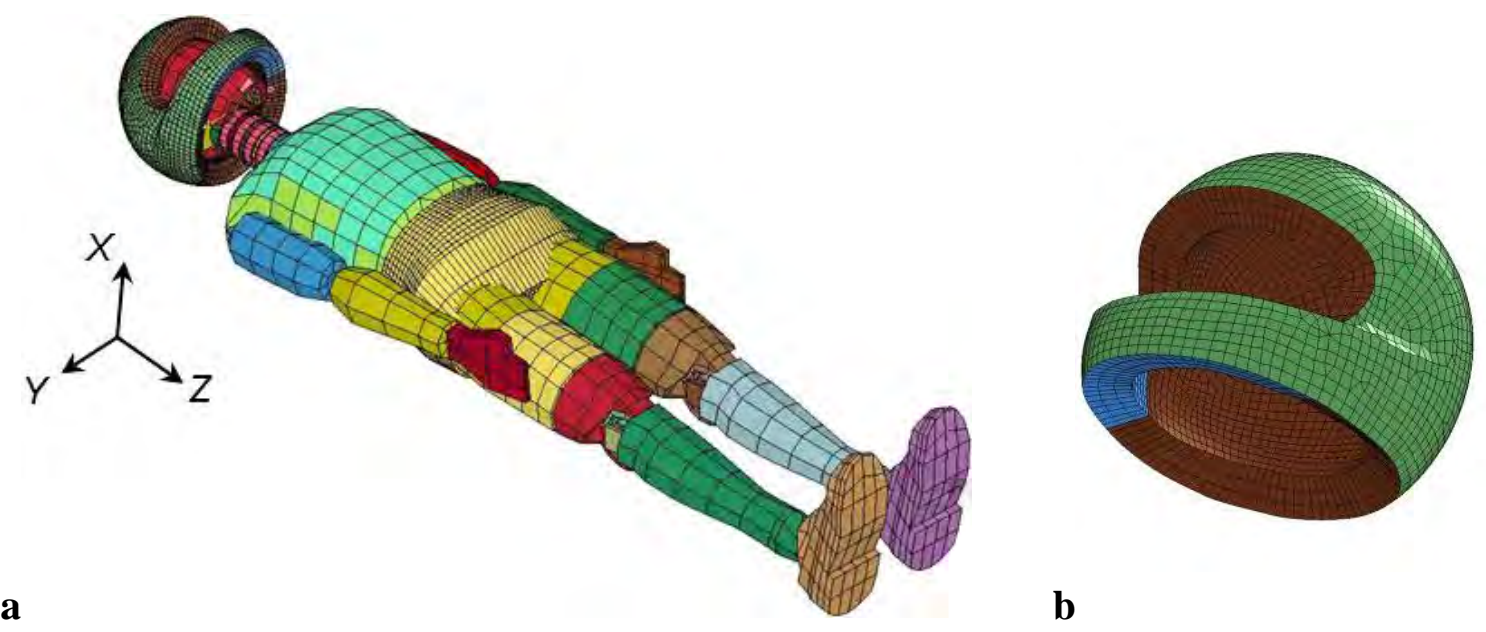

Figure 2: a) The modified dummy used in the virtual tests, b) detail of finite element model of the helmet.

Table II: Materials used in the helmet finite element model.

\begin{tabular}{|l|c|c|c|}
\hline \multicolumn{1}{|c|}{ Part } & Material model & Density $\left(\mathrm{kg} / \mathrm{m}^{3}\right)$ & Young's modulus (MPa) \\
\hline Outer Shell & Plastic Kinematic & 1050 & 1650 \\
\hline Protective padding & Crushable Foam & 40 & 26.5 \\
\hline Comfort padding & Low Density Foam & & 5 \\
\hline
\end{tabular}

The finite element model of the roadside motorcyclist protective device developed in this paper includes detailed representations of all components to ensure that the model was an 
accurate representation of the actual system (Fig. 3). The system modelled consists of two superimposed metal barriers. The upper barrier is the standard W-beam guardrail for vehicle safety, whose geometry and dimensions are listed in the standard. The lower barrier is specially designed to motorcyclist protection. In the barrier, the length of the rails (upper and lower) is of $4315 \mathrm{~mm}$ and are joined together in the area of post, spaced them 4 meters. The upper W-profile rail and the post are connected by means of one M16 $\times 40$ bolt, a washer and nut with a support bracket interposed between them; while the different modules of the rail are jointed together by eight M16 $\times 30$ bolts, 8 washers and 8 nuts. The M16 $\times 40$ bolt attach to the support bracket of the arm, which support the lower barrier. This barrier is attached to the next by six M16×30 bolts and to the arm with a M16 $\times 40$ bolt. In the middle of each barrier, there is an arm that connects the lower rail with the upper one. The support brackets and post are connected by means of three M16x35 bolts, three washers and three nuts.

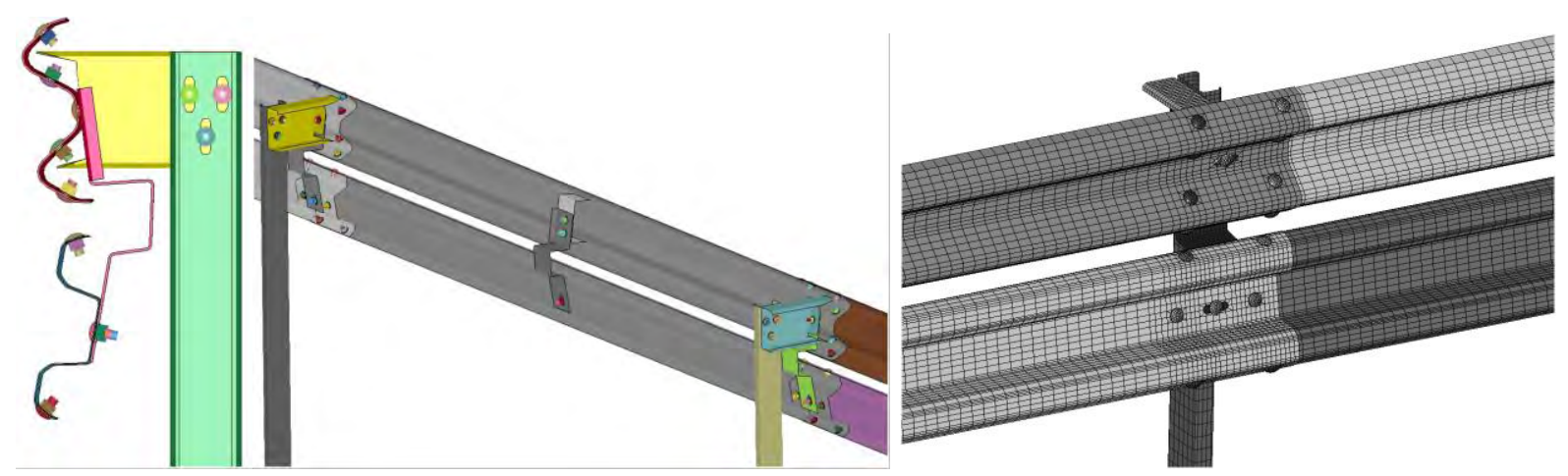

Figure 3: Detailed of the modelled system and the MPS mesh.

All components were modelled using quadrilateral shell elements. The shell element used in this analysis is based on the Belytschko-Lin-Tsay shell formulation. The steel of barriers, posts, support brackets and linkage arms was modelled using an elasto-plastic material. The carbon steel has $300 \mathrm{MPa}$ of yield stress and the behaviour in the plastic field is defined by means of a stress-plastic strain curve. All the bolted connections were modelled in the same way. A rigid material was used in bolts, nuts and washers and the tight force (preload of bolted connection) and tension resistance of the union was modelled by mean of a discrete element of a spring nonlinear elastic material. A contact between the three elements was defined to allow the axial movement.

The soil was modelled as a cylindrical block using eight node hexahedral solid elements. In the centre of the cylindrical block, the shape of the post was incorporated into the soil mesh in order to have representation of the post-soil interaction, without penetration between elements. The posts, with $1500 \mathrm{~mm}$ in length, have $855 \mathrm{~mm}$ under the ground, inside the solid elements. A surface to surface contact was defined between the solid elements of the soil and the shell elements of the post. To represent the soil behaviours, a SOIL_AND_FOAM material model in LS DYNA was used.

\subsection{Model validation}

Thanks to a private company, the test reports of full-scale crash tests were used (centred post and centred rail impacts). The head accelerations and neck forces and moments were used to compare the test and simulations results. A set of initial crash simulations served to identify the influence of different parameters in the model behaviour.

Due to the material properties of different elements were obtained from individual test or from other sources, the unknown parameters in the model are the friction in the contact dummy-soil and dummy-barrier elements and the precise point of impact. The material 
models are previously indicated, and the characteristic parameters are considered as constant in each simulation. There are several contact types and contact-related parameters in LSDYNA that can be used to modify or, in many cases, improve contact behaviour [21]. The contact parameters, in the surface-to-surface contact model, are considered constant along every simulation, but the vales are obtained from validation tasks, after a multi-objective optimization process. The considered contact parameters are: Contact Sliding Friction (Static and Dynamic), Contact Thickness, Viscous Damping, Maximum Penetration, and other geometric and mesh considerations. Therefore, it was necessary to check their influence on the results of the simulation [24]. It is noted that both the friction in contact helmet-barrier as the contact dummy-barrier have significant influence in the path of the dummy and the forces experienced by the neck after the first collision with the barrier. The point of impact has a significant influence on the values of force and acceleration at the instant of initial contact. Therefore, the model was fitted in two phases. In first phase, the point of impact was fitted. In second phase the value of the frictions in dummy contacts were fitted. This procedure is able to reduce setup time, since the simulation to define the collision point does not exceed $10 \mathrm{~ms}$.

In both cases, a multi-objective optimization algorithm (NSGA-II) was used. The objectives are to minimize the mean square error of neck forces and accelerations of the head between test and simulation. An application was developed in order to generate the file for LS-DYNA simulation (Fig. 4).

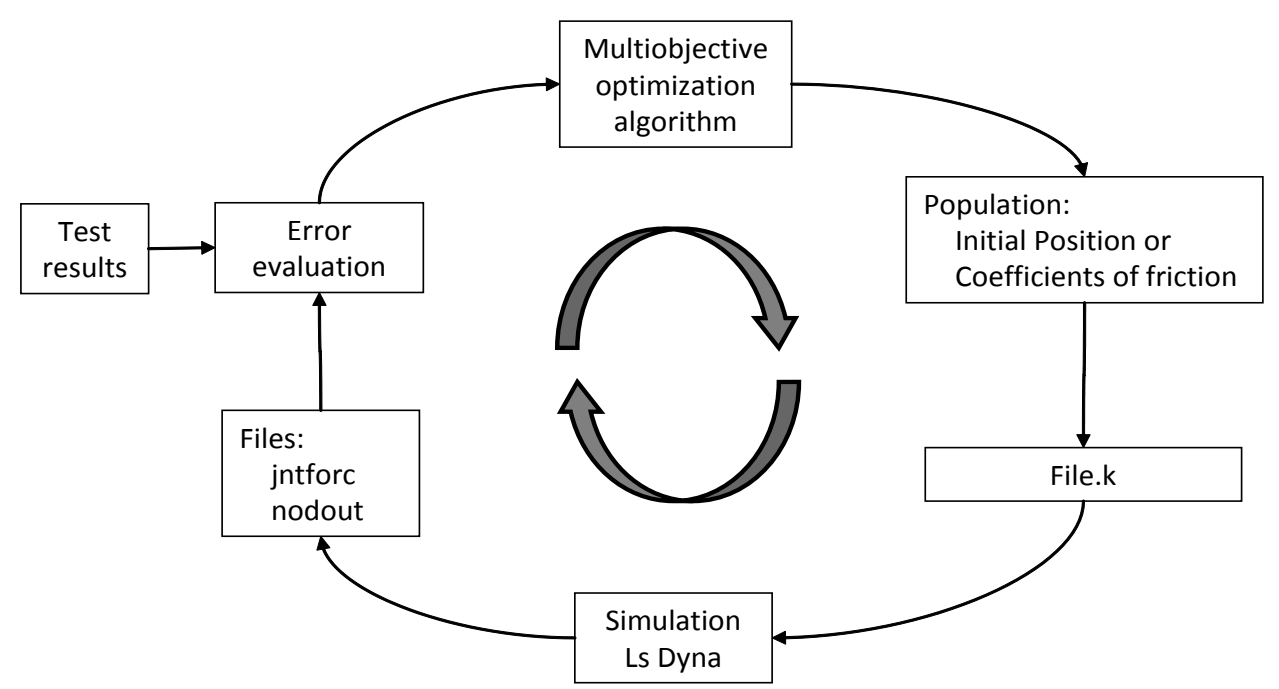

Figure 4: Schematic representation of the fitting procedure.

For example, for the first fitting phase, this application modified the $\mathrm{x}, \mathrm{y}$ and $\mathrm{z}$ coordinates of the nodes of barrier system. The multiobjective algorithm generates a population of two variables. These variables are the horizontal and vertical displacement. The initial position is coincident with the nominal indicate in regulation. The original file (barrierv0.k) is modified in every iteration with these variables, and a new file is written in the same folder (barrier.k) which contains the master file (asembly.k) and the other subfile (dummy.k). The simulation results are written in two files. The application reads these files and calculates the mean square error between simulation results and full-scale crash test results. The multiobjective optimization algorithm uses the calculated errors to generate de following population. As an example, the result of the fitting process can be seen in Fig. 5, where the $Z$ direction neck forces of the test and simulation are represented. 


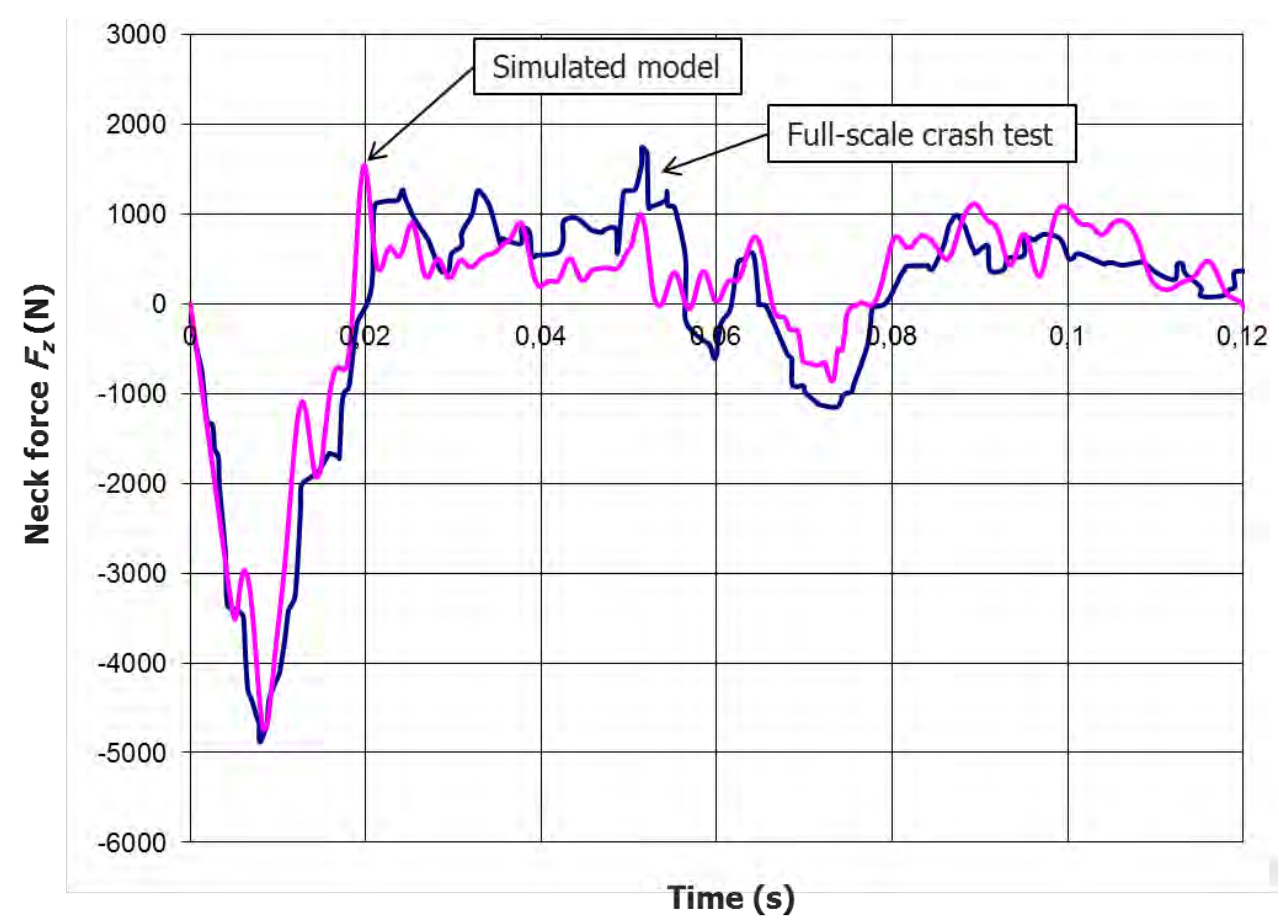

Figure 5: Neck force in $Z$ direction from the post full-scale crash test and simulated model.

\subsection{Virtual tests}

In order to evaluate the influence of impact point height, the set of simulations has been performed, following the standard protocol, at different initial heights of the dummy head. (Fig. 6). During the fitting process, the impact height of the dummy is determined. The result is that the distance obtained from the lowest point of the helmet to the ground was $25 \mathrm{~mm}$ (Fig. 7 a). Taking as reference this height, simulations were performed with different heights, starting at the lowest possible position, $25 \mathrm{~mm}$ below the reference (helmet sliding on the ground) up to $20 \mathrm{~mm}$ above the reference height. Seven different heights were simulated, $10 \mathrm{~mm}, 15 \mathrm{~mm}, 20 \mathrm{~mm}$ and $25 \mathrm{~mm}$ below the reference and $10 \mathrm{~mm}$ and $20 \mathrm{~mm}$ above the reference.

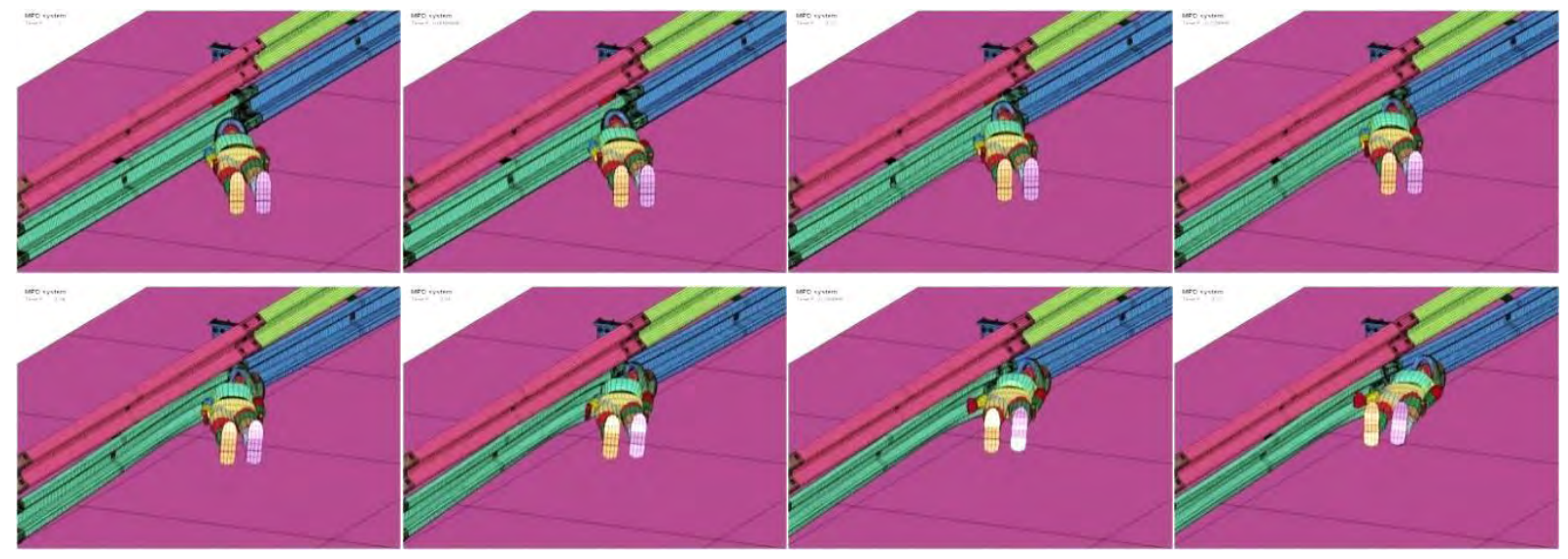

Figure 6: Perspective view of the sequence of the reference impact virtual test - validated finite element model. 

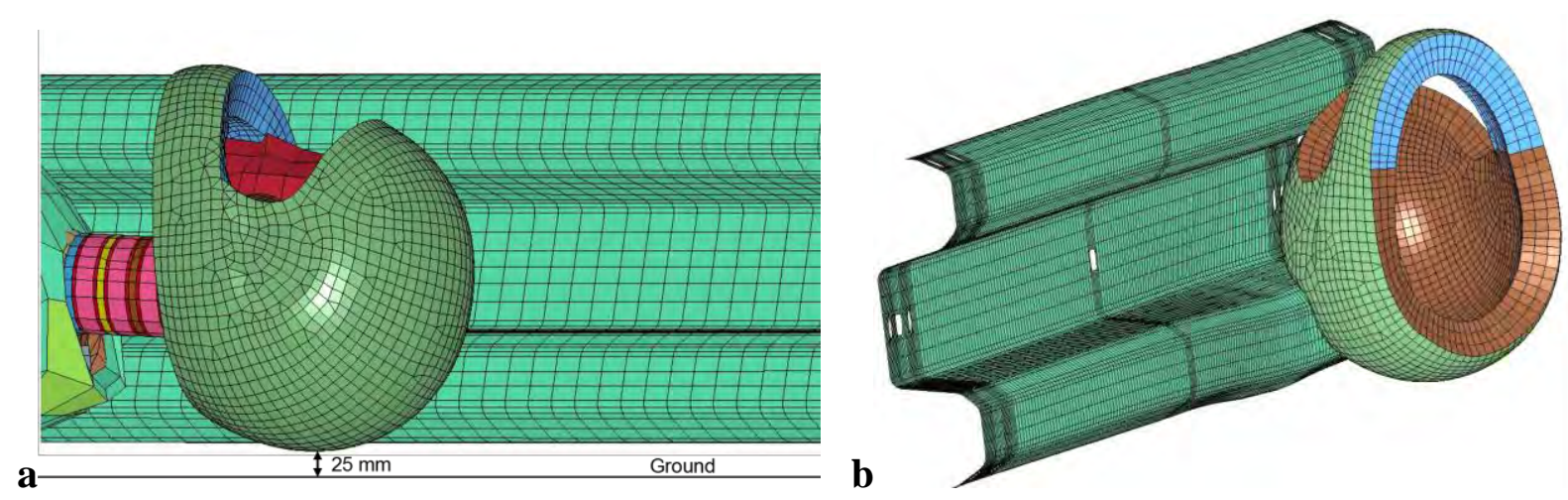

Figure 7: a) The reference impact height of the dummy from the full-scale crash test, b) side view of point of impact.

\section{RESULTS AND DISCUSSION}

In order to evaluate the influence of impact point height the dummy neck forces, the dummy neck moments and the head impact criteria were calculated. After the analysis, the compression neck force ( $Z$ direction) and the neck flexion moment were the most sensitive parameters to the height variation. If the maximum compression neck forces are represented versus the impact height, the Fig. $8 \mathrm{a}$ is obtained. If the maximum force is greater than $4000 \mathrm{~N}$ the system is refused. The maximum compression force for level I is $3200 \mathrm{~N}$.
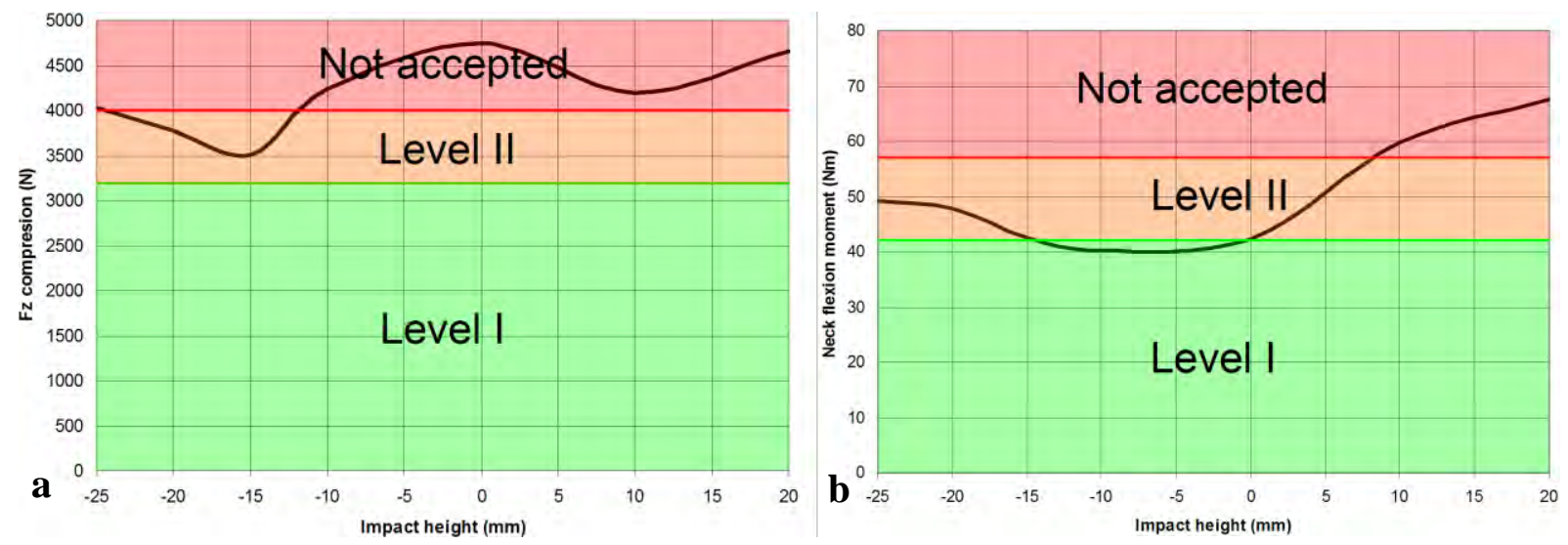

Figure 8: a) The maximum compression neck force, and b) maximum neck flexion moment vs. the impact height.

The maximum compression force is produced in the first impact between the helmet and the motorcyclist protection barrier. In Fig. 7 b, that represents only the helmet and the MPS, can be seen that the impact takes place in a zone of the barrier where there is a significant variation of the geometry with height. This variation of the geometry results in a variation of the impact stiffness, thus justifying the modification of the maximum force on impact.

In the same way, the impact height affect the neck flexion moment (Fig. 8 b). The barrier geometry variations affect the forces and movements of the head during the collision, resulting in a variable bending moment. So this system, due to the compression neck forces, the minimum class of severity that can be obtained is level II, and only in a small range of impact height. In the reference test, the system was refused due to this force but if the impact had taken place in the range of heights above, the system had been accepted. The other variables have not significant variation with the impact height, at least not affect the class of severity. 


\section{CONCLUSION}

The safety devices to protect motorcyclist must be compliant with technical specification. The testing protocol of the European Technical Specification TS 1317-8 simulates a specific type of impact with defined parameters (speed, angle of approach, dummy position, etc.). However, after examining high-speed video recordings of real tests, an important (and close to random) deviation was noticed in the height point of impact due to the geometry of the dummy and helmet and characteristics of sliding surface (loose gravel). When the dummy is moving towards the MPS, the head bounces and rebounds, and the neck changes it angular position. This deviation in angular position modifies the height of impact point. This parameter affects to the contact geometry and is supposed that could have a relevant role in the crash mechanics and injuries causation. The existing testing protocols have no mention to deviation limits or acceptable intervals.

In order to develop a parametric study of the standard testing protocol, a full model of the testing facility and dummy was developed, including helmet and MPS. The virtual model has been designed and implemented to reproduce the tests of the European standard. This numerical model was implemented in computer simulation software (LS-DYNA) to perform a mathematical analysis of virtual tests using the Finite Element Method. The implemented model has been validated and adjusted thanks to the results of an experimental program, which ensures the validity and accuracy of the virtual simulations.

As a result of the parametric study, in this paper the influence of the height of impact point was presented. Deviation limits of $-25 \mathrm{~mm}$ and $+20 \mathrm{~mm}$ from the reference height (according to validation test) of the impact point were analysed. Under this operating conditions, can be asserted that a wide spread of results can be achieved after the testing program. The impact height is in relation with the contact geometry between the helmet and the MPS (which results in a variation of the stiffness) and has a significant influence on loads (forces and moments) in the neck of the dummy. The potential injuries caused by the crash can vary substantially in different points into the considered interval. It has been proved that there is a significant variation of certain indexes (HIC, Forces, ...) when the height of the dummy approach varies, so that, according to this, the level of severity changes. The influence is so important that it could involve rejection or acceptance of a system.

In the analysed MPS, only if a narrow deviation of the impact height $(0-10 \mathrm{~mm}$ over the ground) is considered, the global result of the test (level II) could be the same. The European specification TS 1317-8 do specify any limit deviation interval of the impact height. This height varies due to the procedure itself, so a test cannot be repeated for this reason. The absence of this requirement can reject systems that for other impact height could be valid. Otherwise, the standard can accept systems that optimal working height is reduced and that in case of impact at different heights of the test would be not accepted. This calls into question the objectivity of the standards.

Therefore, the impact height deviation must be limited in the standard testing protocol. To establish an objective and acceptable value of the limit deviation of the impact height, further analysis must be put forward considering other MPSs.

Other influencing parameter not properly defined in mentioned standard are, for instance, the type of soil or its behaviour (affected by humidity for example), the friction coefficient between dummy and soil, etc. They should be included in future works to improve the testing procedure and guarantee the repeatability of the tests.

As a conclusion of the presented work, compliance with the requirements of the standard test does not guarantee that the system is safe under conditions that are not exactly those of the test. This means that it could also be necessary to include other tests. This goal must be analysed deeply so it will be developed in future works. Despite this, a proposed solution is 
develop a test like the head impact test used in vehicle test or a pendulum testing, in order to verify the behaviour of the system at different impact heights. An alternative to the real test is the possibility to introduce and use the "Virtual Testing" concept in the regulation. They also can be used to complete the full test in order to evaluate the performance of safety devices in different impact condition, such as the variation of the impact height.

\section{ACKNOWLEDGEMENTS}

Thanks to the company Mieres Tubos S.L., because without the results of the full-scale crash test and other information provide the validation of the simulation model could have not been possible. Thanks to a project funded by the Spanish Ministy of Education (ref. TRA2006-14429), title: "Metodología para la optimización y ajuste de modelos matemáticos de colisión de vehículos aplicable a la investigación de accidentes de tráfico para la evaluación y mejora de los sistemas de seguridad" this research has been possible.

\section{REFERENCES}

[1] Chang, H. L.; Yeh, T. H. (2006). Risk factors to driver fatalities in single-vehicle crashes: Comparisons between non-motorcycle drivers and motorcyclists, Journal of Transportation Engineering, Vol. 132, No. 3, 227-236, doi:10.1061/(ASCE)0733-947X(2006)132:3(227)

[2] Haque, M. M.; Chin, H. C.; Debnath, A. K. (2012). An investigation on multi-vehicle motorcycle crashes using log-linear models, Safety Science, Vol. 50, No. 2, 352-362, doi:10.1016/j.ssci. $\underline{2011.09 .015}$

[3] ACEM, 2004. MAIDS In depth investigations of accidents involving powered two wheelers, Final Report, from http://ec.europa.eu/transport/roadsafety_library/publications, accessed on 2104-2014

[4] McCarthy, M. G.; Walker, L. K.; Hutchins, R.; Tong, R.; Keigan, M. (2007). Comparative analysis of motorcycle accident data from OTS and MAIDS, Project report, TRL limited, from http://www.maids-study.eu/pdf/OTS MAIDS comparison.pdf, accessed on 21-04-2014

[5] Mooi, H. G.; Galliano, F. (2001). Dutch in-depth accident investigation: first experiences and analysis results for motorcycles and mopeds, SAE Paper Number: 2001-06-0070

[6] Teoh, E. R.; Campbell, M. (2010). Role of motorcycle type in fatal motorcycle crashes, Journal of Safety Research, Vol. 41, No. 6, 507-512, doi:10.1016/j.jsr.2010.10.005

[7] Daniello, A.; Gabler, H. C. (2011). Fatality risk in motorcycle collisions with roadside objects in the United States, Accident Analysis \& Prevention, Vol. 43, No. 3, 1167-1170, doi:10.1016/ j.aap.2010.12.027

[8] Gabler, H. C. (2007). The risk of fatality in motorcycle crashes with roadside barriers, Proceedings of the $20^{\text {th }}$ International Technical Conference on the Enhanced Safety of Vehicles, Lyon, Paper Number 07-0474, 5 pages

[9] Holdridge, J. M.; Shankar, V. N.; Ulfarsson, G. F. (2005). The crash severity impacts of fixed roadside objects, Journal of Safety Research, Vol. 36, No. 2, 139-147, doi:10.1016/j.jsr. $\underline{2004.12 .005}$

[10] Ibitoye, A. B.; Hamouda, A. M. S.; Wong, S. V.; Radin, R. S. (2006). Simulation of motorcyclist's kinematics during impact with W-Beam guardrail, Advances in Engineering Software, Vol. 37, No. 1, 56-61, doi:10.1016/j.advengsoft.2004.12.002

[11] Jama, H. H.; Grzebieta, R. H.; Friswell, R.; McIntosh, A. S. (2011). Characteristics of fatal motorcycle crashes into roadside safety barriers in Australia and New Zealand, Accident Analysis \& Prevention, Vol. 43, No. 3, 652-660, doi:10.1016/j.aap.2010.10.008

[12] Bambach, M. R.; Grzebieta, R. H.; McIntosh, A. S. (2012). Injury typology of fatal motorcycle collisions with roadside barriers in Australia and New Zealand, Accident Analysis \& Prevention, Vol. 49, 253-260, doi:10.1016/j.aap.2011.06.016

[13] Molinero, A. (2009). APROSYS SP 4, Final report for the work on Motorcyclist Accidents (SP4), from http://www.transport-research.info/Upload/Documents/201203/20120313_144753_ 24930_Final SP4 report AP-90-0004.pdf, accessed on 21-04-2014 
[14] García, J.; García, D.; Molinero, A., Perandones, J. M.; Fernández, J. A.; Martín, C.; Mansilla, A. (2009). Improving motorcyclists' safety in Spain by enhanced crash test procedures and implementation guidelines, Proceedings of the $21^{\text {st }}$ International Technical Conference on the Enhanced Safety of Vehicles, Stuttgart, Paper Number 09-0194, 11 pages

[15] Peldschus, S.; Schuller, E.; Koenig, J., Gaertner, M.; García, D.; Mansilla, A. (2007). Technical bases for the development of a test standard for impacts of powered two-wheelers on roadside barriers, Proceedings of the $20^{\text {th }}$ Enhanced Safety of Vehicles Conference, Lyon, Paper Number 07-0332, 13 pages

[16] García, D. R.; Pérez Magallón, B.; Peldschus, S.; Schuller, E.; Mansilla Gallo, A.; Bidal, S. (2010). Overview on the development of a test standard for the evaluation of motorcyclists' impacts on road infrastructure elements, International Journal of Crashworthiness, Vol. 15, No. 1, 1-15, doi:10.1080/13588260902956070

[17] AENOR (2008). Spanish standard UNE-135900-2008: Standard on the evaluation of performance of the protection systems for motorcyclists on safety barriers and parapets

[18] CEN (Centre Européen de Normalisation) (2012). Road restraint systems - Part 8: Motorcycle road restraint systems which reduce the impact severity of motorcyclist collisions with safety barriers, CEN/TS 1317-8:2012

[19] Capitani, R.; Pellari, S. S. (2012). Analysis of the behaviour of biker protection devices for roadside barriers, International Journal of Crashworthiness, Vol. 17, No. 5, 461-478, doi: $10.1080 / 13588265.2012 .678108$

[20] Berg, F. A.; Rucker, P.; Gartner, M.; Konig, J.; Grzebieta, R., Zou, R. (2005). Motorcycle impacts into roadside barriers - real-world accident studies, crash tests, and simulations carried out in Germany and Australia, Proceedings of the $19^{\text {th }}$ International Conference on Enhanced Safety of Vehicles, Washington, DC, Paper Number 05-0095, 13 pages

[21] LSTC (2014). LS-DYNA, http://www.lstc.com/

[22] Toma, M.; Njilie, F. E. A.; Ghajari, M.; Galvanetto, U. (2010). Assessing motorcycle crashrelated head injuries using finite element simulation, International Journal of Simulation Modelling, Vol. 9, No. 3, 143-151, doi:10.2507/IJSIMM09(3)3.164

[23] Arashmehr, J.; Rahimi, G. H.; Rasouli, S. F. (2013). An experimental and numerical investigation of a grid composite cylindrical shell subjected to transverse loading, Strojniski vestnik - Journal of Mechanical Engineering, Vol. 59, No. 12, 755-762, doi:10.5545/sv-jme.2013.1106

[24] Trzepiecinski, T.; Lemu, H. G. (2014). Frictional conditions of AA5251 aluminium alloy sheets using drawbead simulator tests and numerical methods, Strojniski vestnik - Journal of Mechanical Engineering, Vol. 60, No. 1, 51-60, doi:10.5545/sv-jme.2013.1310 